\title{
USANDO NOÇÕES DE BELEZA PARA RECORDAR E SER CONHECIDO NA REGIÃO DO GOLFO DE BENIM
}

\author{
Adedoyin Teriba (Universidade de Princeton/EUA) \\ Tradução para o português: Bárbara Lima \\ Revisão técnica: Roberto Conduru
}

Este artigo estuda como a arquitetura de um grupo de afro-brasileiros retornados ao Golfo do Benim, na virada do século XX, consolida um conjunto de fatores que caracterizaram o seu retorno à África. Primeiro, foi como os afro-brasileiros retornados remodelaram suas identidades sociais. Em segundo, como a arquitetura encarna suas filiações simultâneas a diferentes religiões. Em terceiro lugar era como suas concepções de beleza reforçaram as suas novas identidades sociais e múltiplas filiações religiosas.

\section{ARQUITETURA AFRO-BRASILEIRA; GOLFO DO BENIM; SÉCULO XIX ; SÉCULO XX.}

TERIBA, Adedoyin. Usando noções de beleza para recordar e ser conhecido na região do Golfo de Benim. Textos escolhidos de cultura e arte populares, Rio de Janeiro, v.10, n.1, p. 19-29, mai. 2013. 


\section{USING NOTIONS OF BEAUTY \\ TO REMEMBER \& BE KNOWN IN THE BIGHT \\ OF BENIN AND ITS HINTERLAND}

Adedoyin Teriba (Princeton University/USA)

This article studies how the architecture of a group of Afro-Brazilian returnees to the Bight of Benin at the turn of the 20th century was an embodiment of a constellation of factors that were characteristic of their return. First was how the Afro-Brazilians' returnees re-shaped their social identities. Second was how the architecture embodied their simultaneous affiliations to different religions. Third was how their re-conceptions of beauty reinforced their new social identities and multiple religious affiliations.

AFRO-BRAZILIAN ARCHITECTURE; BIGHT OF BENIN; XIX CENTURY; XX CENTURY.

TERIBA, Adedoyin. Usando noções de beleza para recordar e ser conhecido na região do Golfo de Benim. Textos escolhidos de cultura e arte populares, Rio de Janeiro, v.10, n.1, p. 19-29, mai. 2013. 


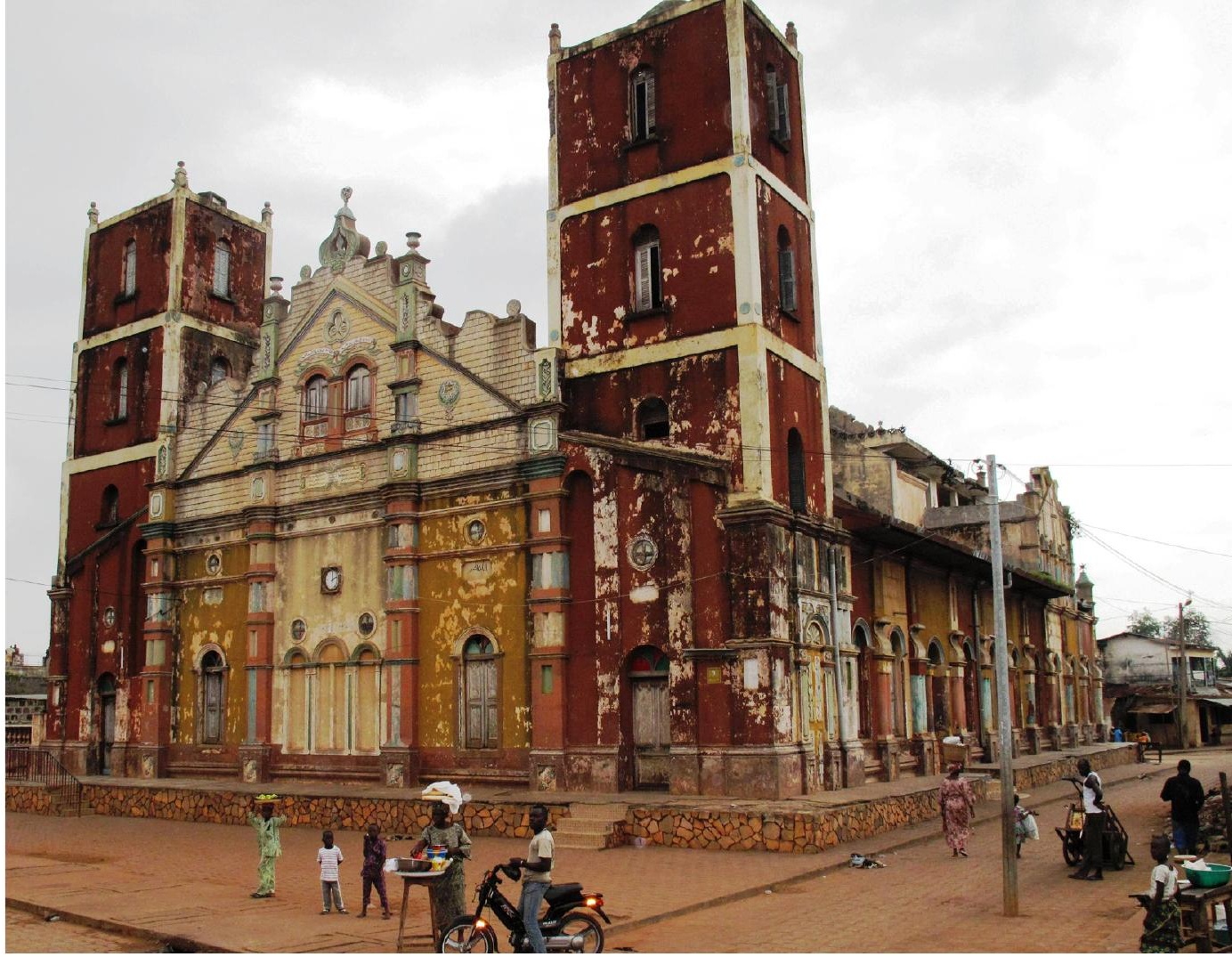

Figura 1: Grande Mesquita em Porto Novo Benim Foto Milton Guran

O comércio transatlântico de escravos acelerou a troca comercial de objetos e pessoas entre as Américas, Europa e o Golfo de Benim entre os séculos XV e XIX. De 1830 em diante, famílias de escravos libertos e expulsos voltaram de navio para o Golfo de Benim e seus arredores, tornando-se funcionários públicos e comerciantes ambulantes nas emergentes colônias europeias. Chegaram do Brasil, Cuba, Santa Helena, Estados Unidos da América, Reino Unido, Jamaica, França, Trinidad e Tobago e Antígua, e constituíram pequena porcentagem da população em seus locais de destino. Em 1861, por exemplo, a colônia britânica de Lagos tinha 2.500 imigrantes em uma população total que excedia 30.000 pessoas (BRoWN, 1964, p. 37). Os nativos do Golfo de Benim os nomearam com palavras recorrentes ouvidas por acaso nas conversas dos próprios imigrantes: aguda, amaro, nagô, saro, aku e ta bom. ${ }^{1}$

Os imigrantes afro-brasileiros perceberam que as formas arquitetônicas tinham que ter integridade, proporção e clareza, ideias estéticas que influenciaram o design de casas e igrejas durante séculos no Brasil. A Catedral de Santa Cruz, a Mesquita Shitta Bey, o Mausoléu do Chefe Taiwo Olowo, a Mesquita Central e a Casa Ebun em Lagos (construídos em 1881, 1894, 1901 e as duas últimas em 1913, respectivamente), bem como a Grande Mesquita em Porto-Novo (Figura 1) e a Mesquita Abomey, ambas construídas em 1913, são exemplos desse ideal. 


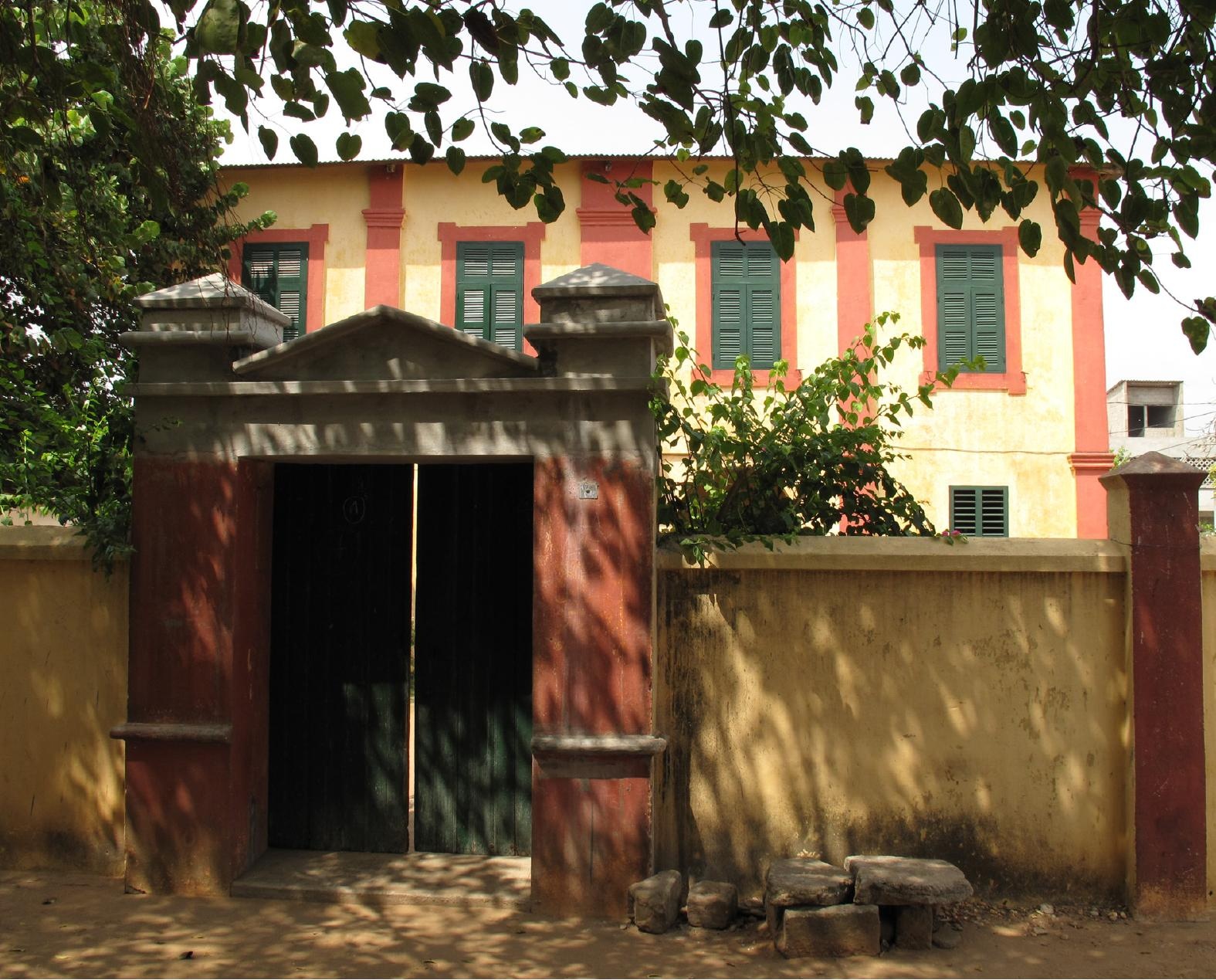

Figura 2: Casa em Agoue, Benim Foto Milton Guran

Entre 1843 e 1856 o sultão otomano Abdulmecid encomendou duas mesquitas neobarrocas em Constantinopla: a Mesquita Ortakoy e a Mesquita Kucuk Mecidiye. Seu neto, o sultão Abdul Hamid II, mais tarde agraciou Mohammed Shitta, financiador da Mesquita Bey Shitta em Lagos, com o título árabe honorífico "Bey" e uma medalha da Ordem Terceira do Medjidiye por construir o que um jornal local chamou "o melhor exemplo de arquitetura eclesiástica na África ocidental". ${ }^{2}$ A medalha era o mais alto título honorífico que o sultão poderia conceder a um civil no Império Otomano. A comunidade muçulmana de Lagos havia informado ao sultão Abdul Hamid II sobre a mesquita vários meses antes de sua conclusão, e ele enviou a medalha por intermédio de William Abdullah Quilliam, seu conselheiro nas Ilhas Britânicas, que discursou na cerimônia de inauguração da mesquita (SINGLETON, 2009, p. 381; EUBA, 1972-1974, p. 13). A Mesquita Shitta Bey e a Mesquita Central em Lagos, bem como a Grande Mesquita e a Mesquita Abomey, em Porto-Novo e Abomey, respectivamente, mostram que as mesquitas 


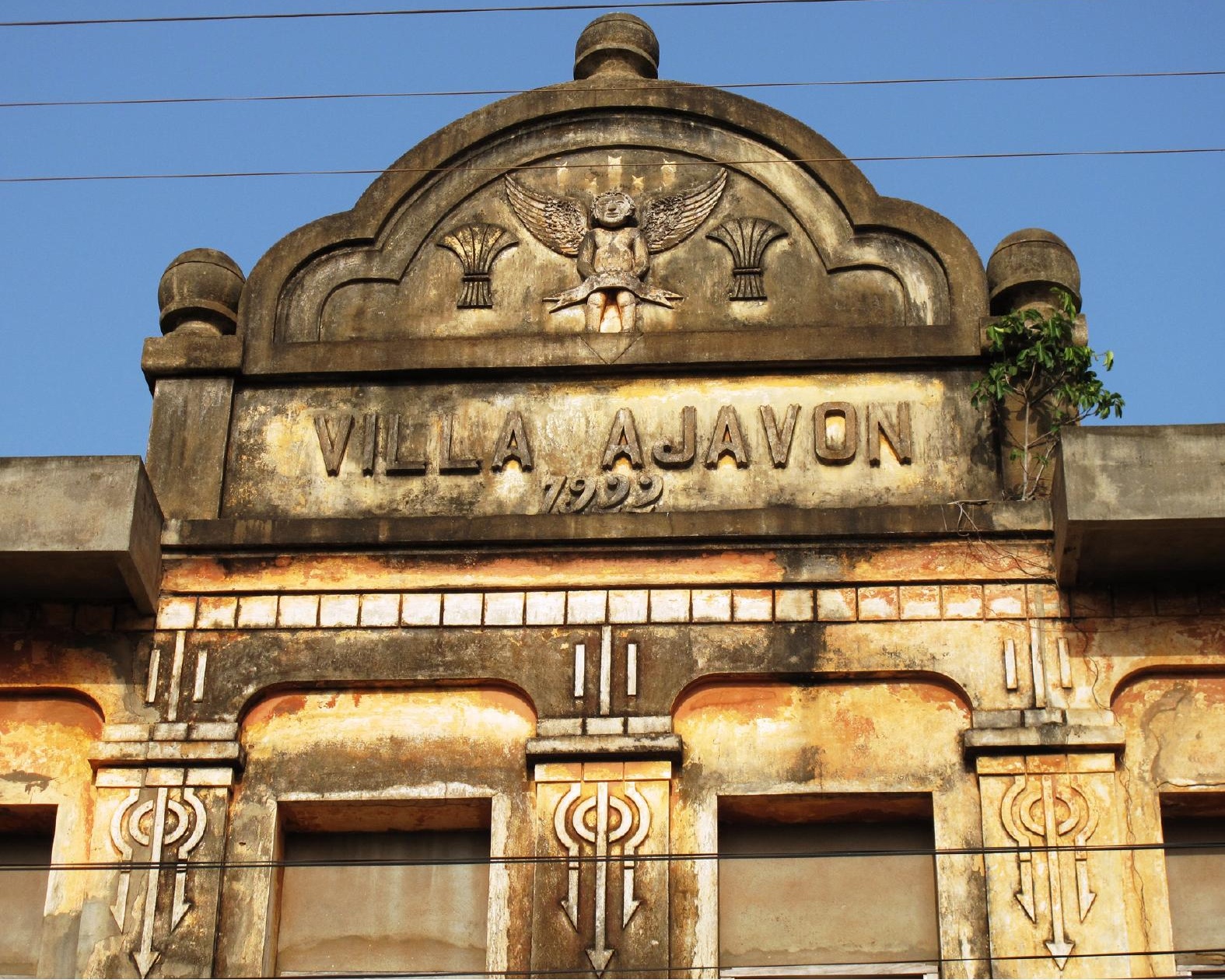

Figura 3: Casa Ajavon 1909 em Ouidah, Benim Foto Milton Guran

neobarrocas existiam em áreas além de Constantinopla, no século XIX e início do XX. Igualmente fascinante é a perspectiva de que as formas arquitetônicas dessas mesquitas no Golfo de Benim surgiram de correspondência constante entre o Sultanato de Constantinopla e comunidades muçulmanas no Golfo de Benim. Isso, no entanto, não explica o emprego, por parte dos imigrantes afro-brasileiros, de uma linguagem arquitetônica intimamente ligada à arquitetura islâmica eclesiástica, e se faz necessário especular por que isso ocorreu. Em primeiro lugar, os construtores imigrantes afro-brasileiros criaram uma arquitetura que ressaltava sua formação artesanal, adquirida de várias formas no Brasil. Alguns eram ex-escravos que haviam trabalhado nas missões jesuíticas (MENEZES, 1988, p. 25), apesar de sua prática secreta do islamismo e de religiões autóctones, mesmo após sua recepção na Igreja católica. A exposição dos escravos a elementos expressivos da fé católica em procissões, missas e batismos pode justificar em parte a utilização de elementos de ritual cristão em suas mesquitas. Em segundo lugar, essa 
apropriação era parte de uma grande liberdade artística que caracterizou a cultura visual cristã, islâmica e do candomblé dos afro-brasileiros, que se apropriaram de elementos uns dos outros. Consequentemente, os afro-brasileiros que praticaram essas religiões simultaneamente favoreceram certos dogmas de uma das religiões em detrimento de outros. ${ }^{3}$

Martiniano do Bonfim, natural de Salvador, cujos pais foram levados como escravos do sudoeste da Nigéria, era carpinteiro e ajudou a construir a Catedral de Santa Cruz, em Lagos, já mencionada (TURNER, 1942, p. 59-61; ${ }^{4}$ MATORY, 2005, p. 46-63). Era também adivinho e introduziu inovações litúrgicas nas cerimônias de candomblé em muitos templos em Salvador e talvez tenha construído outro em Ilaro, cidade perto de Lagos. O artesão muçulmano Manoel Friandes construiu vários edifícios para as autoridades católicas, incluindo uma igreja na Lapinha, em Salvador, na década de 1860. Decorou as paredes da nave com arcos "árabes" e uma inscrição em árabe significando "Eis que Este é o Milagre de Deus e esta é a Porta do Céu" (GoMEZ, 2005, p. 127). Assim, as atividades de Bonfim e Friandes são exemplos concretos do intercâmbio transcultural que personificava a arte das três religiões mais populares praticadas pelos afro-brasileiros.

Nem todos os construtores imigrantes, no entanto, haviam sido escravos. Alguns, como Aleijadinho, escultor e arquiteto mulato brasileiro do século XVIII, eram filhos de antigos escravos que aprenderam ofícios com os pais. Além disso, arquitetos portugueses no Brasil asseguraram a seus filhos mulatos o ensino privado do conhecimento de escultura e arquitetura. A Missão Jesuítica do Colégio Santo Alexandre, no Estado do Pará, no Brasil, treinou muitos escravos que ensinaram a outros afro-brasileiros livres em áreas urbanas ao longo dos séculos XVIII e XIX (MENEZES, 1988). Outros artesãos foram alunos de escolas de artesanato que surgiram no Brasil no século XIX. A Escola de Arte e Artesanato da Bahia, inaugurada em Salvador em 1872, ofereceu aulas noturnas gratuitas destinadas a criar uma força de trabalho para construção, composta de indivíduos livres que aos poucos pudesse substituir o trabalho manual do escravo soteropolitano. Graduar-se em uma instituição desse porte pode ter elevado psicologicamente os afro-brasileiros que tinham pouco acesso a oportunidades econômicas em uma época que só lenta e tardiamente viu, de fato, a abolição da escravatura (HAWTHORNE, 2010). Em Salvador, esses artesãos nascidos livres se reuniram em associações denominadas Cantos, que se encontravam em esquinas e compravam projetos de construção, posteriormente distribuídos entre seus membros (REIS, 1993, p. 164).

Muitos afro-brasileiros migraram para as periferias das cidades no Brasil e no Golfo de Benim, na África Ocidental, em parte devido às escassas oportunida- 


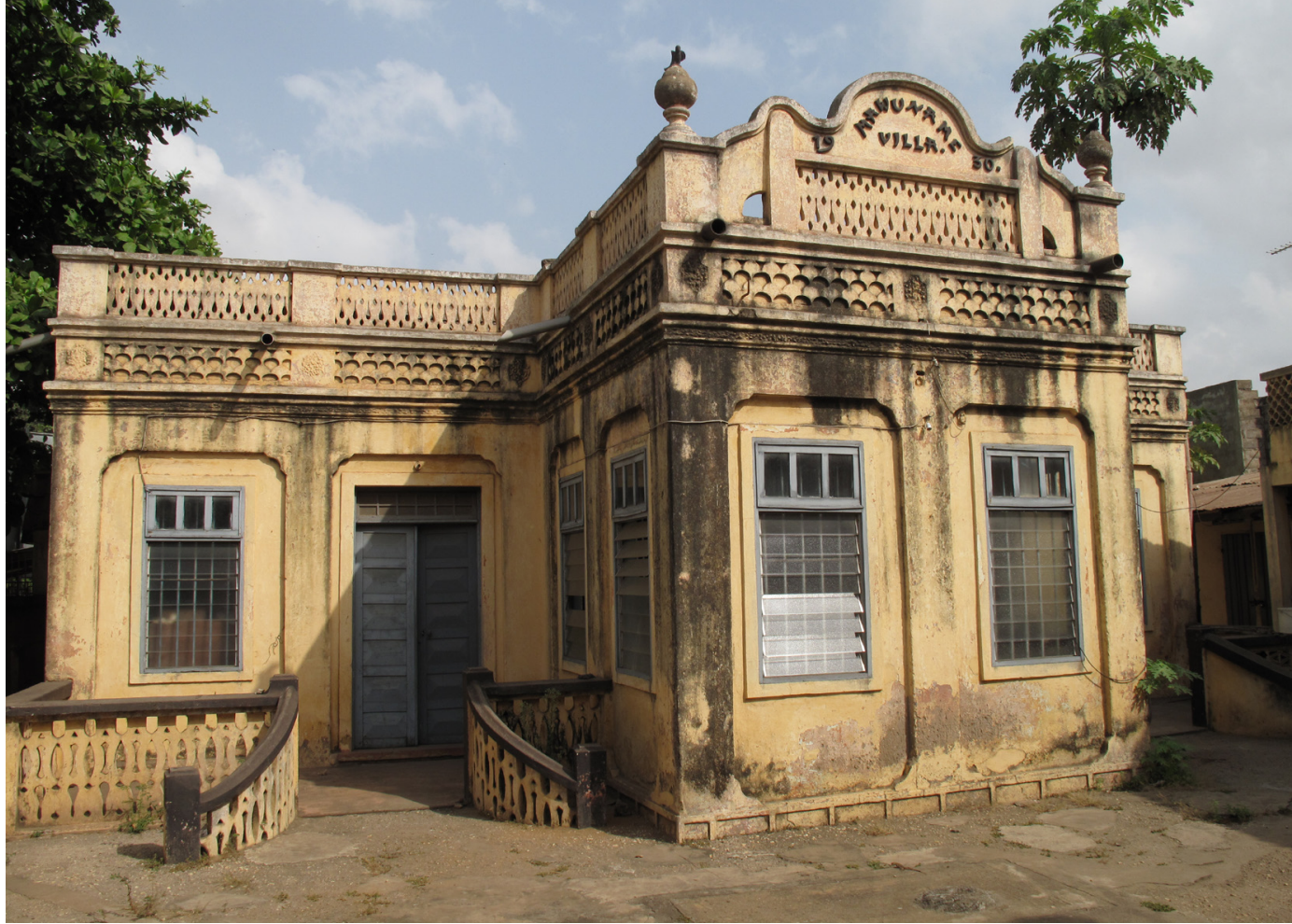

Figura 4: Villa Mawuname em Lome, Togo Foto Milton Guran

des econômicas nos centros urbanos. O governo brasileiro também expulsou os instigadores dos tumultos que assolaram a cidade de Salvador ao longo do século XIX, enviando-os para o Golfo de Benim, particularmente depois da revolta dos Malé, em 1835 (VERGER, 1968). ${ }^{5}$

Os artesãos imigrantes afro-brasileiros estabeleceram rapidamente reputação como o grupo de artesãos mais qualificado em todo o Golfo de Benim e seus arredores, e criaram uma arquitetura que reforçava essa posição no Golfo. Eles também queriam que os edifícios afirmassem a superioridade moral e intelectual do grupo sobre os profissionais locais. Mesquitas, igrejas, mausoléus, templos de orixás, palácios e casas (figuras 2 a 4) eram feitos de terra, pedra e tijolo, revestidos com azulejos de cerâmica e tinham disposição clássica idiossincrática, padrões florais em baixo-relevo, esculturas heráldicas, bem como a estrela de davi e a lua crescente islâmica. ${ }^{6} \mathrm{~A}$ realeza, os sacerdotes das religiões locais e os leigos nas cidades nigerianas de Badagry, Oshogbo e Ekiti construíram arquitetura semelhante nas décadas subsequentes, introduzindo novos elementos e descartando outros. Os novos elementos foram sacadas, balaustradas e muros muito decorados, bem como esculturas heráldicas abstratas.

A arquitetura dos imigrantes afro-brasileiros e das gerações subsequentes no Golfo de Benim revelou gradual transformação dos critérios de beleza que refletiam suas identidades em processo. Essa metamorfose foi baseada na cren- 
ça de que a arquitetura era capaz de comunicar que os imigrantes eram, em suas palavras, mais bonitos do que a população local. Beleza, aos olhos dos imigrantes afro-brasileiros, dos monarcas locais, dos chefes e dos comerciantes, era conceito que conjugava ideais estéticos e políticos, e ajudou a unir o que, aos olhos dos nativos, constituía comunidades diferentes. Esse grupo variado de pessoas implantou certos elementos arquitetônicos para colonizar informalmente a população local descrevendo o impacto cultural que a arquitetura de um grupo de imigrantes brasileiros exerceu em várias cidades no Golfo de Benim. Esse impacto foi análogo, em alguns aspectos, à doutrinação cultural experimentada pelos colonizados em territórios coloniais (LAOTAN, 1943, p. 1). ${ }^{7}$ Os elementos visuais das construções foram criados para reforçar a autopromoção das identidades dos grupos.

A migração dos afro-brasileiros para o Golfo de Benim foi única por várias razões. Primeiro, ela destacou a forma como alguns desses imigrantes se estabeleceram no Golfo, mesmo que tenham sido levados como escravos de outras partes da África (BROWN, 1964, p. 34). Talvez sua arquitetura até mesmo os tenha ajudado a romper a ligação anterior com suas terras de origem, declarando que os novos assentamentos eram seu verdadeiro lar. Além disso, os imigrantes podem ter tentado refazer sua difícil permanência no exterior como escravos ou indivíduos economicamente marginalizados em narrativa segundo a qual, em último caso, prosperaram como cidadãos no Brasil. Seus prédios ricamente ornamentados, assim, simbolizavam essa narrativa e cimentavam as fluidas identidades locais de imigrantes afro-brasileiros, resultantes da tentativa da população nativa de entender quem eram os imigrantes quando ouviam seus falares.

\section{REFERÊNCIAS BIBLIOGRÁFICAS}

AKINSEMOYIN, Kunle; VAUGHN-RICHARDS, Alan. Edifício Lagos. Lagos: F \& A Services, 1976.

BENEDETTI, Carla De. Moschee D’Africa, Domus, n. 658, p.30-37. 1985.

BROWN, Spencer H. A History of the People of Lagos State, 1852-1886. Dissertação, Northwestern University, Evanston, 1964.

CARROLL, Kevin. Architectures of Nigeria: Architectures of the Hausa and Yoruba Peoples and of the Many Peoples between Tradition and Modernization. London: Ethnographica Limited, 1992.

CUNHA, Marianno Carneiro da. Das senzalas para as casas de cidade: arquitetura brasileira na Nigéria e República Popular do Benin. São Paulo: Nobel, 1985.

DMOCHOWSKI, Zbigniew. An Introduction to Nigerian Traditional Architecture. London: Ethnographica, 1980. 
DREWAL, Henry. Memory and Agency: Bantu and Yoruba Arts in Brazilian Culture. In: Diaspora and Visual Culture: Representing Africans and Jews. London: Routledge, 2000.

EUBA, Titilola. Shitta Bey and the Lagos Muslim Community 1850-1895. Nigerian Journal of Islam 2, n. 2, p. 13. 1972-1974.

FYLE, C. Magbaily. The Yoruba Diaspora in Sierra Leone's Krio Society. In: FALOLA, Toyin; CHILDS, Matt D. (eds.). The Yoruba Diaspora in the Atlantic World. Bloomington: Indiana University Press, 2004.

GOMEZ, Michael A. Black Crescent: The Experience and Legacy of African Muslims in the Americas. Cambridge: Cambridge Univeristy Press, 2005.

HALLEN, Barry. Afro-Brazilian Mosques in West Africa. Mimar: Architecture in Development, n. 29, p. 16-23, 1988.

HAWTHORNE, Walter. From Africa to Brazil: Culture, Identity, and an Atlantic Slave Trade, 1600-1830. New York: Cambridge University Press, 2010.

KOYTOFF, Jean. A Preface to Modern Nigeria. Madison: University of Wisconsin Press, 1965.

LAOTAN, A.B. The Torch Bearers or Old Brazilian Colony in Lagos. Lagos: Ife-Olu Printing Works, 1943.

LAW, Robin. Yoruba Slaves Who Returned to West Africa In: FALOLA, Toyin; CHILDS Matt D. (eds.). The Yoruba Diaspora in the Atlantic World. Bloomington: Indiana University Press, 2004.

MACMILLAN, Alister (ed.). The Red Book of West Africa. London: Frank Cass and Company Ltd.,1968.

MATORY, J. Lorand. Black Atlantic Religion: Tradition, Transnationalism and Matriarchy in the Afro-Brazilian Candomble. Princeton: Princeton University Press, 2005.

MARAFATTO, Massimo. Brazilian Houses Nigeriane $=$ Nigerian Brazilian Houses. Lagos: Instituto Italiano di Cultura, 1983.

MENEZES, José Luis Mota. The Presence of Blacks and Mulattoes in Pernambuco Art, In: DRYSDALE, Eric (ed.). The Afro-Brazilian Touch: The Meaning of Its Artistic and Historic Contribution. São Paulo: Tenenge, 1988.

OLINTO, Antonio. Water House. New York: Caroll and Graf Publishers, 1985.

REIS, João José. Slave Rebellion in Brazil: The Muslim Uprising of 1835 in Bahia. Baltimore: Johns Hopkins University Press, 1993.

SCHAUMLOEFFEL, Marco Aurelio. Tabom. The Afro-Brazilian Community in Ghana. Bridgetown: Schaumloeffel Editor/Custom Books Publishing, 2009.

SINGLETON, Brent D. That Ye May Know Each Other: Late Victorian Interactions between British and West African Muslims. Journal of Muslim Minority Affairs, v. 29, n. 3, p. 381, 2009. 
SOUMONNI, Elisée. The Afro-Brazilian Communities of Ouidah and Lagos in the Nineteenth Century: A Comparative Analysis. In: CURTO, Jose C.; SOULOUDRE-LA FRANCE, Renée (eds.). Africa and the Americas: Interconnections During the Slave Trade. Trenton: Africa World Press, 2005.

TURNER, Lorenzo. Some Contacts of Brazilian Ex-Slaves with Nigeria, West Africa, Journal of Negro History, v. 27, n. 1, p. 59-61, 1942.

VERGER, Pierre. Trade Relations between the Bight of Benin and Bahia from the 17th Century to the 19th Century. Ibadan: University of Ibadan Press, 1968.

\section{NOTAS}

1 Musibau Akanni, diretor da Casa da Nigéria, o centro cultural da Nigéria em Salvador, Brasil, disse-me que "aguda" é uma corruptela de "Rua d'Ajuda", onde os ex-escravos moravam em Salvador. Robin Law (2004, p. 350) declarou que os daomeanos usavam o termo para distinguir os imigrantes afro-brasileiros dos imigrantes portugueses. "amaro" originou-se de termo usado para designar as áreas dos mercadores estrangeiros no sudoeste da Nigéria. O grupo étnico fon no Daomé chamava o povo anagô do sudoeste da Nigéria contemporânea "nagô" (MATORY, 2005, p. 4). Matory atribui a grande atenção que a cultura nagô recebeu na historiografia da escravidão brasileira do século XIX a divulgadores que escreveram histórias nagô em livros ingleses que foram amplamente divulgados. Os "professores de inglês" classificaram largamente a civilização nagô como iorubá (MATORY, 2005, p. 46-64). Os Krio, em Serra Leoa, chamavam os escravos libertos do sudoeste da Nigéria de "aku", termo derivado de ekusheh (bem feito em iorubá), e os residentes em Lagos os denominaram saros, quando eles retornaram (FYLE, 2004, p. 369 е KOYTOFF, 1965, p. 86). Os habitantes de Gana chamavam os imigrantes afro-brasileiros de "ta bom", que significa "está bom" em português do Brasil. Alguns tabom mudaram-se de Lagos para Accra (SCHAUMLOEFFEL, 2009).

2 "The Consecration of Mr. Shitta's Mosque", Lagos Weekly Record, 7 de julho de 1894.

3 Os imigrantes afro-brasileiros muçulmanos do sexo masculino, na sua maioria, ao contrário de suas contrapartes locais, se casaram (soumonNI, 2005, p. 238).

4 Em notas de rodapé, Turner cita Bonfim como seu informante.

5 Verger afirmou que nove revoltas ocorreram entre 1807 e 1835 na Bahia.

6 Para descrições de alguns poucos arquitetos e construtores, ver Macmillan (1968, p. 106, 108, 110, 114); Laotan (1943, p. 7, 11-17); Gomez (2005, p. 127) e Drewal (2000, pp. 243-244). Lázaro Borges da Silva, Francisco No- 
bre e Baltazar dos Reis trabalharam em muitos projetos juntos. Para imagens e ilustrações de sua arquitetura na Nigéria e República do Benim, ver Cunha (1985, p. 100-169); Akinsemoyin e Vaughn-Richards (1976, p 16-28); Hallen (1988, p. 16-23); Benedetti (1985, p. 30-37); Carroll (1992, p. 74-78); Dmochowski (1980, p. 2-56) e Marafatto (1983).

70 título do livro evidencia que os Laotan viam a comunidade afro-brasileira em Lagos como uma colônia brasileira. Ver também Olinto (1985, p. 77). Em um dos casos mais reveladores da relação entre os imigrantes afro-brasileiros e os residentes locais de Lagos, no romance de Olinto, uma mulher aguda se gabou de que os afro-brasileiros ensinaram aos moradores numerosos ofícios da construção civil e introduziram sementes de mandioca e castanha de caju no protetorado britânico de Lagos.

Adedoyin Teriba é doutorando de história da arquitetura na Universidade de Princeton nos Estados Unidos da América. Nascido da Nigéria, ele trabalhou como arquiteto e gerente de projeto em seu país de origem e nos Estados Unidos.

Recebido em: 12/05/2012

Aceito em: 28/09/2012 
